\title{
Measuring the Quality of HTTP Video
}

\author{
Shi Zhiming ${ }^{12}$ Huang Chengti ${ }^{12}$ \\ ${ }^{1}$ College of Engineering, Huaqiao University, Quanzhou Fujian 362021, China \\ ${ }^{2}$ Fujian Provincial Academic Engineering Research Centre in Industrial \\ Intellectual Techniques and Systems \\ szmi_2007@126.com
}

\begin{abstract}
HTTP video streaming is widely deployed to deliver stored media. But the HTTP video quality assessment method is uncertain. In this paper we propose an objective assessment based on Mamdani inference. First we introduce three application performance metrics of HTTP video, including the initial buffering time, mean rebuffering duration, rebuffering frequency. Then we give an experiment to change the network environment and test the HTTP video quality. Our ultimate goal is to characterize the correlation between the application performance metrics and the user QoE using Mamdani fuzzy inference. The experimental results show that the method accords closely with human subjective judgment, and improves the similarity.
\end{abstract}

Keywords: HTTP video; application performance metric; fuzzy mathematics method; $Q o E$

\section{Introduction}

With the growing of network video business, HTTP video streaming have been widely used in our life, such as YouTube.[1] This kind of "streaming" is different from the traditional UDP-based streaming, which is not necessary for the video streaming server and client to synchronize. The client can watch an incompletely downloaded video clip by its download technology. So the HTTP video quality will be influenced by the TCP throughput. When the TCP throughput is lower than video bit rate, the video will pause and wait for new video data. [2]

However, the TCP throughput can be reduced by various kinds of impairments in the network paths, such as packet loss rate, delay. These influencing factors could greatly impact the user-perceived quality, which is also known as the quality of experience (QoE). So how to guarantee the network quality of service (QoS), and improve the user QoE, is very important. The QoE is usually expressed by using a Mean Opinion Score (MOS) of 1("Bad") to 5("Excellent"). [3][4] It could be obtained from subjective to objective measurement. ITU-T and VQEG provide detailed test plans for evaluating video quality in a subjective way. Meanwhile, Peak-Signal-to-Noise-Ratio (PSNR), Mean Square Error (MSE) and Structural Similarity Index (SSIM), [5] which are examples of the objective approach, only evaluate the video quality based on UDP protocol, therefore not suitable for HTTP video. Mok, Ricky K.P has done some research on HTTP video quality assessment. He use line regression to establish the relationship between the application QoS and QoE.[10] But the similarity of this method is not high. S.Egger has tested the video quality under different network environment, and established the relationship between the BufferingCount and QoE. He doesn't consider other factors may impair the HTTP video quality.[13] Kamal Deep Singh has proposed an assessment method based on random neural network.[14] This method needs to train the sample. This method may be influenced by training time and training step. 
As shown in figure 1, this paper propose a temporal evaluation of video as the application QoS for HTTP video streaming and use it to analyze how the QoE is affected by the application QoS. Previous works performed analytical studies to model the relationship between the network QoS and application QoS using TCP. [6] Moreover, empirical studies were conducted to investigate how network conditions affect the application QoS by recording application metrics during the video playback. [7]

We adopt both analytical and empirical approaches to study the correlation between the application QoS and User QoE. We use a set of application QoS for study: Initial buffering time, mean duration of a re-buffering event and re-buffering frequency. Then we set different network parameters and test the HTTP video. We measure the application QoS, and 23 people is organized to evaluate the HTTP video under the different network environment. Based on these experiment data, we correlate the relationship between the application QoS and the user QoE using Mamdani fuzzy inference.

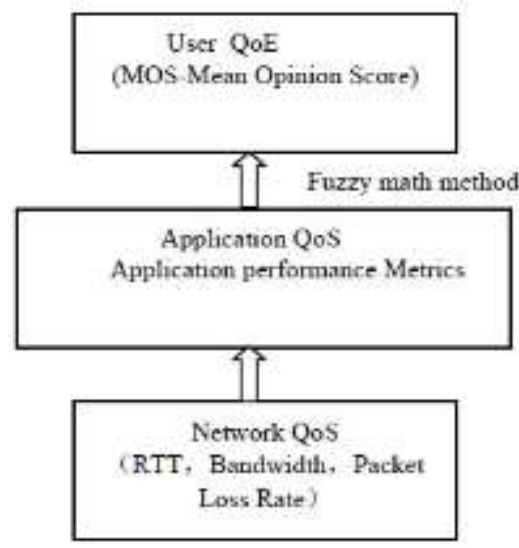

Figure 1. The Relationship between the QoE and the QoS

The main contributions of the paper are: 1 .We set different network environment and test the HTTP video; 2.The relationship between the application QoS and the user QoE is proposed using Mamdani fuzzy inference. The remainder of the paper is organized as follows: Section 2 introduces the three application performance metrics of HTTP video. Section 3 we present the experimental system and measure the video quality under different network environment. Section 4 the principle of fuzzy math and the Mamdani fuzzy inference are introduced. Section 5 we give the relationship between the application QoS and the user QoE using Mamdani fuzzy inference. Section 6 we give the experimental results. This paper is concluded in section 7 .

\section{The Application QoS of HTTP Video}

Previous works on correlating network QoS and User QoE mainly concern picture quality. Fuzheng Yang studied how the packet loss affected the video streaming quality, by extracting the related information from the compressed bit stream.[8] Zhou et al. used the objective approach, E-model and the approach proposed in [9] to measure how the perceived quality of VoIP and MPEG-2 video streaming were affected in IPV6. Ricky K.P.Mok et al. changed different network environment and tested the HTTP video quality [10]. Firstly we introduce three application performance metrics to quantify the application QoS for HTTP video streaming, and these metrics represent the temporal structure of a video playback, regardless of the video content.

\subsection{Initial Buffering Time}

Here we use $T_{\text {init }}$ to represent the Initial buffering time. This metric measures the period between the starting time of loading a video and the starting time of playing it. 


$$
T_{\text {init }}=\frac{B_{\text {full }}^{\prime} l}{b}
$$

Where $B_{\text {full }}$ is the size of the video buffer, ${ }^{l}$ is the video's bitrate(bits/s), and ${ }^{b}$ is the average TCP throughput(bits/s) for the video streaming.

\subsection{Mean Rebuffering Duration}

$T_{\text {rebuf }}$ is used to represent the mean rebuffering duration. This metric measures the average duration of a rebuffering event.The estimate (in seconds) of the mean rebuffering duration can be computed by:

$$
T_{\text {rebuf }}=\left\{\begin{array}{l}
0 \text { if } \beta \geq \lambda \\
\frac{\left(B_{\text {full }}-B_{\text {empty }}\right) \times \lambda}{\beta} \text { otherwise }
\end{array}\right.
$$

Where $B_{\text {empty }}\left(<B_{\text {full }}\right)$ is the remaining length of the buffered video that triggers an empty-buffer event.

\subsection{Rebuffering Frequency}

Here, $f_{\text {rebuf }}$ is used to denote the rebuffering frequency. When the amount of buffered video data decreases to a low value, the playback will pause, and the player will enter into a rebuffering state. The metric measures how frequent the rebuffering events occur. Given a video's length of $l$ (in seconds), the rebuffering frequency estimate is given by:

$$
\begin{gathered}
f_{\text {rebuf }}= \begin{cases}0 & \text { if } \beta \geq \lambda \\
\frac{n_{\text {rebuf }}}{l} & \text { otherwise }\end{cases} \\
n_{\text {rebuf }}=\left[\frac{l^{\prime}}{b_{\text {rebuf }}}\right] \\
l^{\prime}=l-\frac{B_{\text {full }}}{\left(1-\frac{\beta}{\lambda}\right)} \\
b_{\text {rebuf }}=\frac{\left(B_{\text {full }}-B_{\text {empty }}\right)}{\left(1-\frac{\beta}{\lambda}\right)}
\end{gathered}
$$

When the average TCP throughput is less than the video's bitrate, we will encounter $\mathrm{n}_{\text {rebuf }}$ empty-buffer events during the video playback given by Equation (4), where $l$ ' is the remaining length of the video (in seconds) upon the onset of the first empty-buffer event, and brebuf is the length of the played video(in seconds) before the next empty-buffer event.From the equation above, we can see that the TCP throughput influence the application QoS. But TCP throughput is decided by the network performance index. 


\section{The HTTP Video Test Experiment}

The experimental system is shown in figure 2 . A web server was installed with Ubuntu 10.04 and Apache 2.4.2 to host video clips for a client to download and play. The switch can control the network bandwidth and the network simulator can emulate different packet loss rates and RTTs.

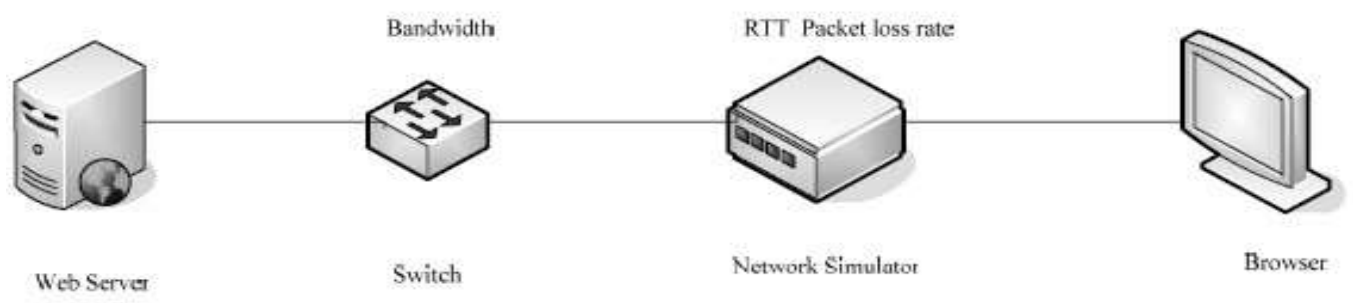

Figure 2. The Experiment System of Video Quality Test

We measure four video contents under different network environment. The video information is recorded in table 1 .

Table 1. The Video Information

\begin{tabular}{|c|c|c|c|c|c|}
\hline Num & Resolution & Bit mate & Video & Content & Time \\
\hline 1 & $1280 \times 544$ & $4.28 \mathrm{Mbps}$ & The Simpsons & $\begin{array}{l}\text { Cartoon } \\
\text { TV }\end{array}$ & 2005 \\
\hline 2 & $640 \times 480$ & $5.16 \mathrm{Mbps}$ & Advertisement & NIKE & 305 \\
\hline 3 & $1920 \times 816$ & $8.23 \mathrm{Mbps}$ & Legend & $\begin{array}{l}\text { Legend } \\
\text { Movie }\end{array}$ & $123 \mathrm{~s}$ \\
\hline 4 & $640 \times 480$ & $10.85 \mathrm{Mbps}$ & Sports & Football & $254 \mathrm{~s}$ \\
\hline
\end{tabular}

Here we use the Simpsons as test example. Table 2 lists the network QoS parameters emulated by the experiment system. The bandwidth was chosen 5Mbps, emulating the bandwidth of common home users. The choices for RTT were chosen between 70ms and $300 \mathrm{~ms}$. We also varied the packet loss rate from $0.1 \%$ to $1 \%$ to investigate the impact of packet loss rate.

Table 2. The Test Network Parameters

\begin{tabular}{|l|lc|}
\hline Bandwidth & \multicolumn{2}{|c|}{$5 \mathrm{Mbps}$} \\
\hline P & $0.1 \%, 0.2 \%, 0.3 \%, 0.4 \%, 0.5 \%, 0.6 \%, 0.7 \%, 0.8 \%, 0.9 \%, 1 \%$ \\
\hline RTT & $70 \mathrm{~ms} \quad 160 \mathrm{~ms} \quad 200 \mathrm{~ms}$ & $300 \mathrm{~ms}$ \\
\hline
\end{tabular}

The browser's cache folder was first cleared, and the video will not be saved to the local cache after quitting the browser. For every set of parameters, 23 users are organized in our experiment; they watch the video from the web server under different network environment. Each user gives the subjective score. We average normalized viewer rating for the same video to determine its MOS[11]. Meanwhile we measure the application metrics of Simpsons video. All the measure results have been averaged. Figure 3 shows the application metrics under different QoS parameters. We can see that the application metrics increase when the $\mathrm{p}$ and RTT increase. All application metrics increase will cause the user wait. 

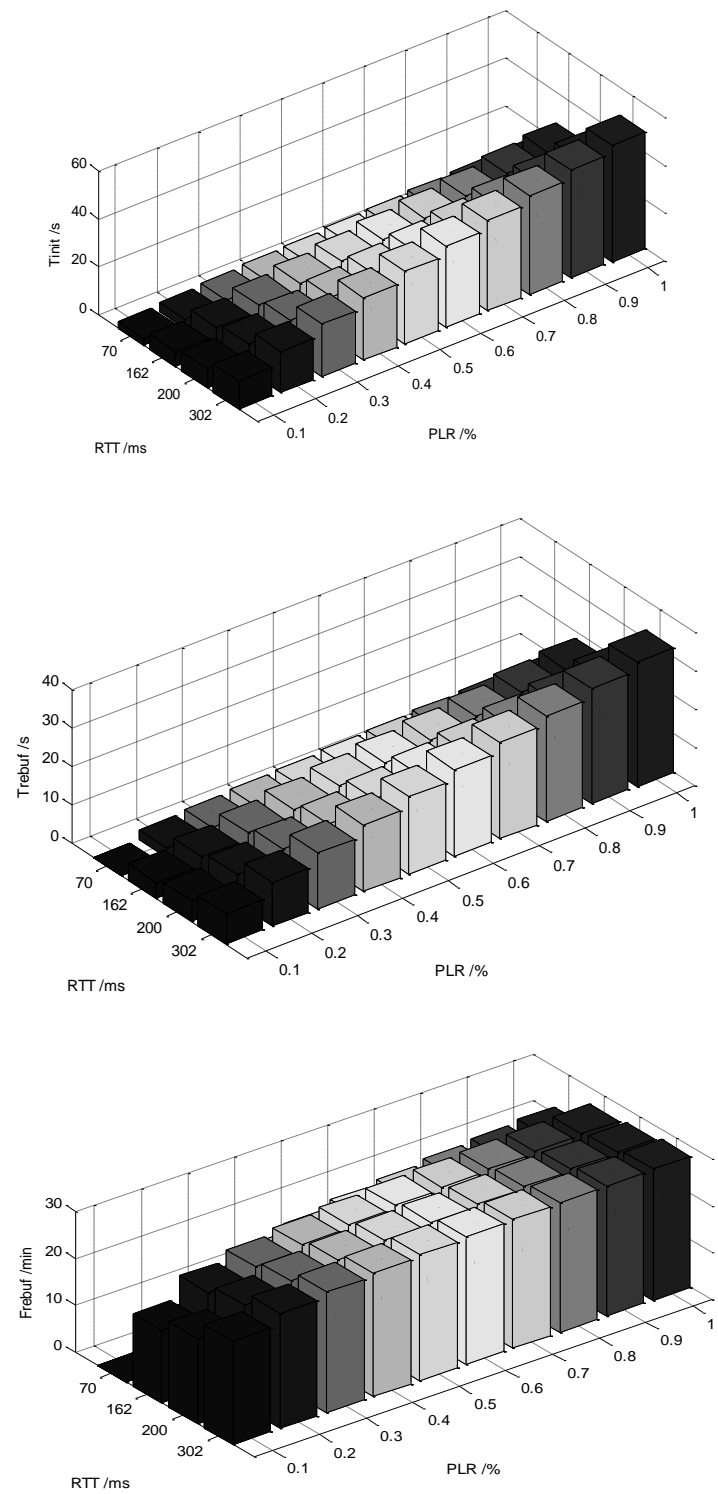

Figure 3. The Applications Performance Metrics

Figure 4 shows the MOS under different QoS parameters. Because the network environment degradation, the MOS decreases. The user waits too much time and cannot watch video. On the other hand, we can see that there exists a relationship between the application metrics and MOS. 


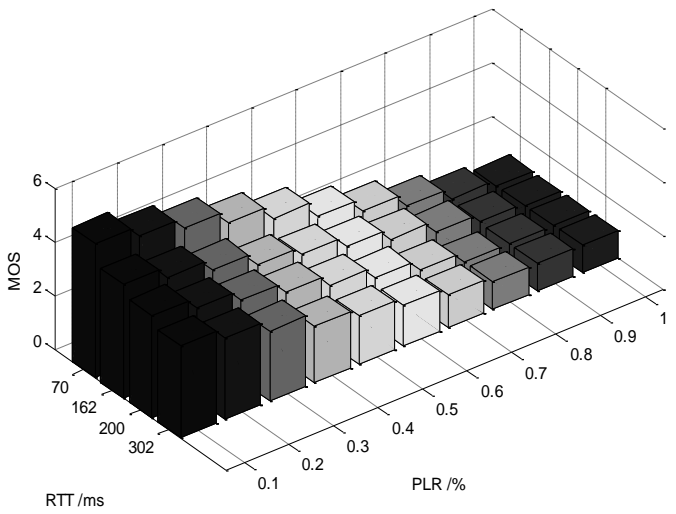

Figure 4. The MOS under Different Network Parameters

\section{The Principle of Mamdani Fuzzy Inference}

In this paper we use fuzzy math method to analyze the relationship between the application performance metrics and MOS. Fuzzy math was proposed by L.A.Zadeh in 1965. It can deal with the fuzziness phenomenon, and the core of fuzzy math mainly depends on the fuzzy rules or inference algorithm. The process of fuzzy math contains four parts: fuzzy interface, knowledge base, fuzzy inference and defuzzification output[12]. Figure 5 shows that the process of fuzzy.

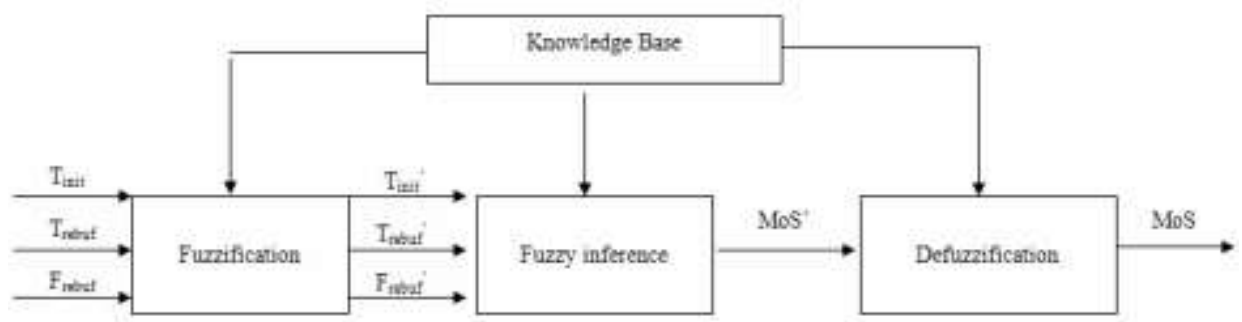

Figure 5. The Fuzzy Math Method

First the input data will be changed into fuzzy hierarchy by fuzzy interface. For example the NB,NM,NS,ZE,PS,PM and PB are the common fuzzy hierarchy. Here NB represents the larger negative, NM represents the general negative, NS represents the small negative, ZE represents zero, PS represents small positive, PM represents the general positive, and $\mathrm{PB}$ represents the larger positive. The knowledge base contains all input and output data, the corresponding theory domain, fuzzy rule table, fuzzy sets. Especially fuzzy rule table can reflect the accurate of the fuzzy model. According to the fuzzy rule table, the fuzzy inference can assess a series of comprehensive problem, in order to get a fuzzy output. Here we introduce the Mamdani fuzzy inference. This inference uses Max-Min inference.

For example the input $\mathrm{x}$ is $\mathrm{A}^{\prime}$, and $\mathrm{y}$ is $\mathrm{B}$ '.

$\mathrm{R} 1$ : if $\mathrm{x}$ is $\mathrm{A} 1$ and $\mathrm{y}$ is $\mathrm{B} 1$ then $\mathrm{z}$ is $\mathrm{C} 1$;

also $\mathrm{R} 2$ : if $\mathrm{x}$ is $\mathrm{A} 2$ and $\mathrm{y}$ is $\mathrm{B} 2$ then $\mathrm{z}$ is $\mathrm{C} 2$;

also $\mathrm{Rn}$ : if $\mathrm{x}$ is $\mathrm{An}$ and $\mathrm{y}$ is $\mathrm{Bn}$ then $\mathrm{z}$ is $\mathrm{Cn}$. 
The Output $\mathrm{z}$ is C'.

The process of Mamdani fuzzy inference can be expressed by

the formula 7,8,9:

$$
\begin{aligned}
C^{\prime} & =\left(A^{\prime} \text { and } B^{\prime}\right) \circ R \\
R & =\bigcup_{i=1}^{n} R_{i} \\
R_{i} & =\left(A_{i} \text { and } B_{i}\right) \rightarrow C_{i}
\end{aligned}
$$

Here contains three fuzzy logic operations: "and" is used to compute intersection; " o " is used to compute Max-Min sets; " $\rightarrow$ " is also used to compute intersection. Defuzzification output can convert the fuzzy domain into precise output. Weighted average method is a common defuzzification method. Formula 10 compute the sum of $\mathrm{m}$ input data, and average them.

$$
z_{0}=\frac{\sum_{k=1}^{m} \mu_{k} C_{k}}{\sum_{k=1}^{m} \mu_{k}}
$$

Here ${ }^{\mu_{k}}$ represents the probability of k, $C_{k}$ represents the value of k.

\section{The Assessment Method}

In this section, we will give the proposed assessment method. The assessment step can be divided into 3 points: 1 . Input application QoS need to be divided into different fuzzy hierarchy. 2 . The fuzzy rule table should be designed. 3 . The assessment method based on Mamdani inference. Now we will describe the three step detail.

\subsection{Fuzzy Hierarchy}

Because the application metrics have been measured 40 times. We divide the input quantity into five grades(NB,NM,ZE,PM,PB). As shown in figure 6, we draw the membership function of Tinit, Trebuf and Frebuf . Here Frebuf has been converted into buffering count per minute. The membership function of Tinit can be expressed.

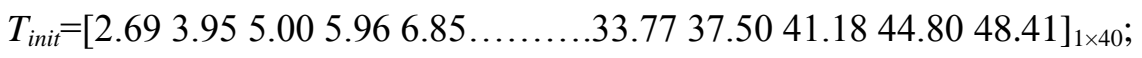

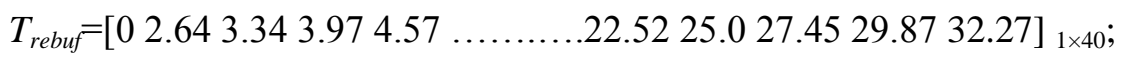

$$
\begin{aligned}
& F_{\text {rebuf }}=\left[\begin{array}{llllllllllll}
0 & 6.80 & 11.60 & 14.40 & 16.40 & 18.0 & \ldots 26.23 & 26.69 & 27.30 & 27.51 & 27.69 & 27.84
\end{array}\right]_{1 \times 40} \text {; } \\
& N B=\frac{0}{2.69}+\ldots+\frac{0.13}{37.50}+\frac{0.39}{41.18}+\frac{0.521}{44.80}+\frac{1}{48.41} \\
& P M=\frac{0}{2.69}+\frac{0.04}{3.95}+\frac{0.186}{5.00}+\frac{0.21}{5.96}+\frac{0.34}{6.85}+\ldots+\frac{0.13}{25.93} \\
& P B=\frac{1.0}{2.69}+\frac{0.88}{3.95}+\frac{0.79}{5.00}+\frac{0.72}{5.96}+\frac{0.62}{6.85}+\ldots+\frac{0.01}{14.57}
\end{aligned}
$$


Here the numerator represents the probability, the denominator represents the input value. In the same way, we can divide the MOS value into five grades. As shown in figure 7, we draw the membership function of MOS. So it can be expressed:

$$
\begin{aligned}
& N B=\frac{1.0}{1.0}+\frac{0.5}{1.5}+\frac{0.0}{2.0}+\frac{0.0}{2.5}+\frac{0.0}{3.0}+\ldots+\frac{0.0}{5.0} \\
& P M=\frac{0.0}{1.0}+\frac{0.0}{1.5}+\frac{0.0}{2.0} \ldots+\frac{0.5}{3.5}+\frac{1.0}{4.0}+\frac{0.5}{4.5}+\frac{0.0}{5.0} \\
& P B=\frac{0.0}{1.0}+\frac{0.0}{1.5}+\frac{0.0}{2.0} \ldots+\frac{0.0}{4.0}+\frac{0.5}{4.5}+\frac{1.0}{5.0}
\end{aligned}
$$

According to the above step, every input variable can find the corresponding probability and judge the status area.
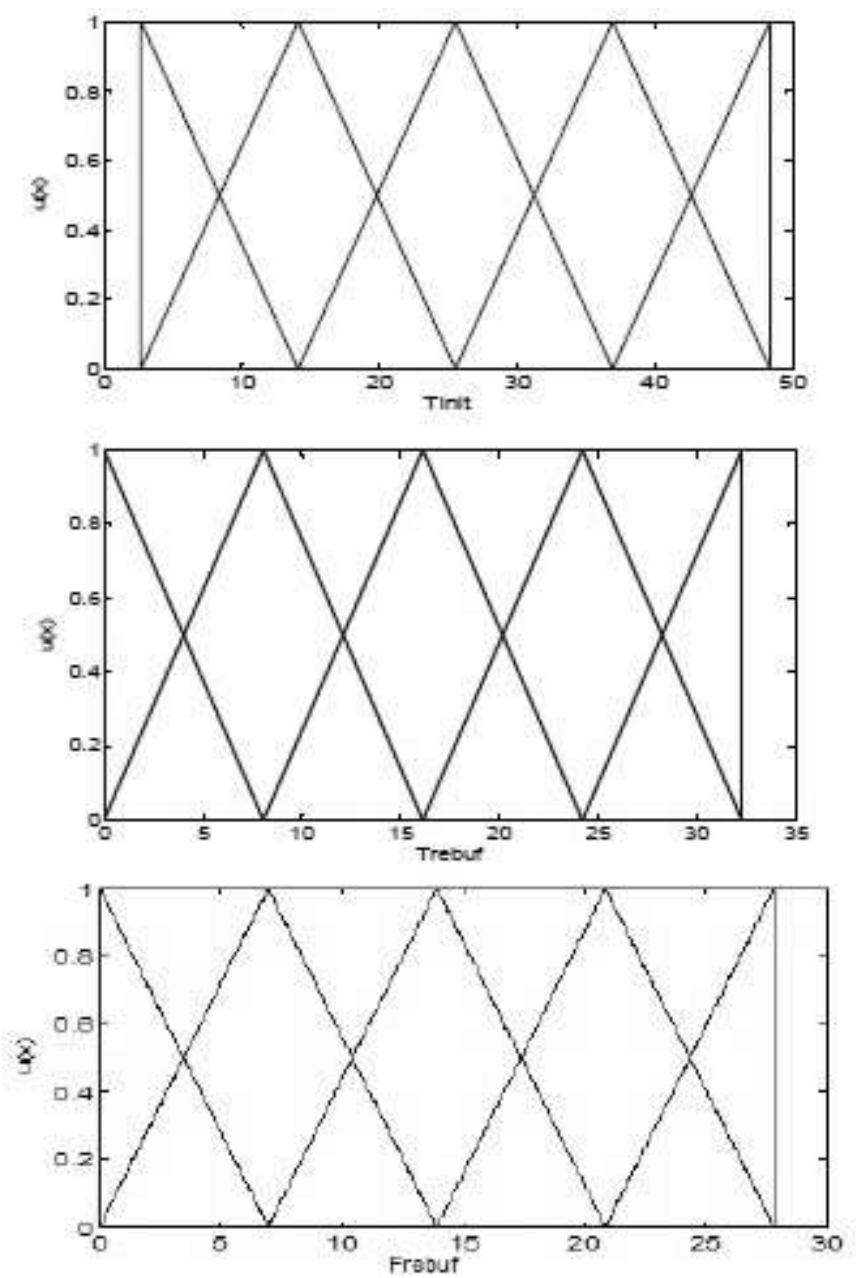

Figure 6. The Membership Function of Application Metrics 


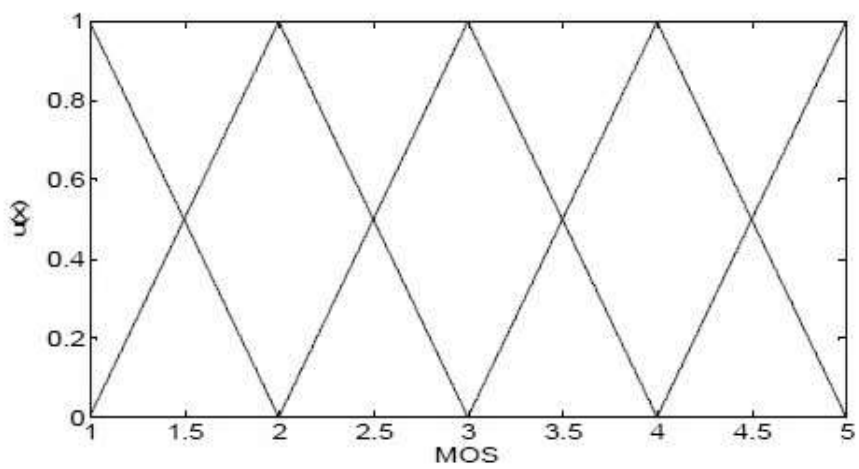

Figure 7. The Membership Function of MOS

\subsection{Fuzzy Rule Table}

According to the user experience, we make the fuzzy rule. It is shown in Table 3. For example, if Frebuf is NB , it represents that the user often waits for the video loading, the Tinit and Trebuf can't be low. So the table III doesn't show the statue of MOS.

The other 45 rules may be appeared.

$\mathrm{R} 1$ : if Frebuf is NB, Tinit is NB and Trebuf is NB, the MOS is NB

R2: if Frebuf is NB, Tinit is NM and Trebuf is NM, the MOS is NM.

R45: if Frebuf is PB, Tinit is $\mathrm{PB}$ and Trebuf is $\mathrm{PB}$, the MOS is $\mathrm{PB}$.

According to the rule table, we can deduce the statue of MOS. We use A represents $T_{\text {init }}$ , B represents $T_{\text {rebuf }}, \mathrm{C}$ represents $F_{\text {rebuf }}$ and D represents MOS. A',B',C'and D' represent the corresponding fuzzy domain.

Table 3. The Test Network Parameters

\begin{tabular}{|c|c|c|c|c|c|c|c|c|c|c|c|c|c|c|c|c|c|c|c|c|c|c|c|c|c|}
\hline \multicolumn{6}{|c|}{$F_{\text {rebuf }} \quad P B$} & \multicolumn{5}{|c|}{$\mathrm{PM}$} & \multicolumn{5}{|c|}{ ZE } & \multicolumn{5}{|c|}{ NM } & \multicolumn{5}{|c|}{ NB } \\
\hline$x=0$ & & $P$ & $\mathrm{Z}$ & $\mathrm{N}$ & $\mathrm{N}$ & $P$ & $P$ & Z & $\mathrm{N}$ & $\mathrm{N}$ & $P$ & $P$ & $\mathrm{z}$ & $\mathrm{N}$ & $\mathrm{N}$ & $P$ & $P$ & $\mathrm{Z}$ & $\mathrm{N}$ & $\mathrm{N}$ & $P$ & $P$ & $\mathrm{Z}$ & $\mathrm{N}$ & $\mathrm{N}$ \\
\hline Not & B & $\mathrm{M}$ & $E$ & M & B & B & $\mathrm{M}$ & E & $\mathrm{M}$ & B & $B$ & $\mathrm{M}$ & $E$ & $\mathrm{M}$ & B & B & $\mathrm{M}$ & E & $\mathrm{M}$ & $B$ & $\mathrm{~B}$ & $\mathrm{M}$ & E & $\mathrm{M}$ & $B$ \\
\hline$P$ & $P$ & $P$ & $\mathrm{P}$ & $/$ & 1 & $P$ & $\mathrm{P}$ & $P$ & 1 & 1 & $P$ & $P$ & $\mathrm{P}$ & $/$ & 7 & $/$ & $/$ & 1 & $/$ & 1 & 1 & 1 & 1 & 1 & 1 \\
\hline B & B & $\mathrm{M}$ & $\mathrm{M}$ & & & B & $\mathrm{M}$ & $\mathrm{M}$ & & & B & $\mathrm{M}$ & $\mathrm{M}$ & & & & & & & & & & & & \\
\hline$P$ & $P$ & $\mathrm{P}$ & $P$ & $/$ & 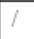 & $P$ & $P$ & Z & 1 & 7 & $P$ & $P$ & $z$ & $/$ & 7 & 1 & $/$ & 1 & $/$ & 1 & 1 & 7 & 1 & / & 1 \\
\hline $\mathrm{M}$ & B & $\mathrm{M}$ & $\mathrm{M}$ & & & B & $\mathrm{M}$ & E & & & $\mathrm{M}$ & $\mathrm{M}$ & E & & & & & & & & & & & & \\
\hline$Z$ & $/$ & 7 & $/$ & $/$ & 1 & $/$ & $\mathrm{P}$ & $Z$ & $z$ & 7 & 1 & $P$ & $\mathrm{Z}$ & $\mathrm{N}$ & 1 & $/$ & $Z$ & $Z$ & $\mathrm{~N}$ & $/$ & 1 & 7 & 1 & 1 & 1 \\
\hline E & & & & & & & $\mathrm{M}$ & E & E & & & $\mathrm{M}$ & E & $\mathrm{M}$ & & & E & E & $\mathrm{M}$ & & & & & & \\
\hline $\mathrm{N}$ & 7 & 7 & $/$ & $/$ & $/$ & 1 & $/ 1$ & $1 /$ & $1 /$ & 7 & $/$ & $/$ & $\mathrm{z}$ & $\mathrm{N}$ & $\mathrm{N}$ & $/$ & 7 & 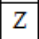 & $\mathrm{N}$ & $\mathrm{N}$ & $/$ & $/$ & $\mathrm{N}$ & $\mathrm{N}$ & $\mathrm{N}$ \\
\hline $\mathrm{M}$ & & & & & & & & & & & & & $E$ & $\mathrm{M}$ & $\mathrm{M}$ & & & E & $\mathrm{M}$ & B & & & $\mathrm{M}$ & $\mathrm{M}$ & B \\
\hline $\mathrm{N}$ & 7 & 7 & $1 /$ & $/ 1$ & $1 /$ & 1 & $1 /$ & $1 /$ & $1 /$ & 7 & 1 & $/$ & $z$ & $\mathrm{~N}$ & $\mathrm{~N}$ & 1 & 1 & $\mathrm{~N}$ & $\mathrm{~N}$ & $\mathrm{~N}$ & 1 & $/$ & $\mathrm{N}$ & $\mathrm{N}$ & $\mathrm{N}$ \\
\hline B & & & & & & & & & & & & & $E$ & $\mathrm{M}$ & B & & & $\mathrm{M}$ & $\mathrm{M}$ & B & & & & $\mathrm{M}$ & B \\
\hline
\end{tabular}

\subsection{Fuzzy Inference Process}

Firstly, every input variable must be changed into fuzzy fields. Here we use formula 11 to change the input variable. Where $x$ represents the input application performance. For example, when $T_{\text {init }}=2.69, T_{\text {rebuf }}=0, F_{\text {rebuf }}=0$, the $\mathrm{A}^{\prime}=\mathrm{B}^{\prime}=\mathrm{C}^{\prime}=\left[\begin{array}{ll}1 & 0 \ldots .\end{array}\right] 1 \times 40 ;$ if $T_{\text {init }}$

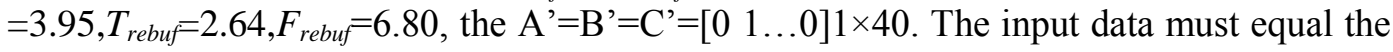
$\mathrm{x} 0$, otherwise the $\mu_{X^{\prime}}(x)=0$. 


$$
\mu_{x^{\prime}}(x)=\left\{\begin{array}{c}
1 \mathrm{x}=\mathrm{x}_{\mathrm{o}} \\
\mathrm{O} \mathrm{x} \neq \mathrm{x}_{\mathrm{o}}
\end{array}\right.
$$

The assessment method uses the Mamdani fuzzy inference. We use formula 12 to deduce the MOS.

$$
\begin{aligned}
D^{\prime} & =\left(A^{\prime} \times B^{\prime} \times C^{\prime}\right) \circ R=\left(A^{\prime} \times B^{\prime} \times C^{\prime}\right) \circ \bigcup_{i=1}^{N} R_{i} \\
& =\bigcup_{i=1}^{N}\left[A^{\prime} \circ R_{i A}\right] \bigcap\left[B^{\prime} \circ R_{i B}\right] \bigcap\left[C^{\prime} \circ R_{i C}\right] \\
& =\bigcup_{i=1}^{N}\left[A^{\prime} \circ\left(A_{i} \rightarrow D_{i}\right)\right] \bigcap\left[B^{\prime} \circ\left(B_{i} \rightarrow D_{i}\right)\right] \bigcap\left[C^{\prime} \circ\left(C_{i} \rightarrow D_{i}\right)\right] \\
& =\bigcup_{i=1}^{N} D_{i A}^{\prime} \bigcap D_{i B}^{\prime} \bigcap D_{i C}^{\prime} \\
& =\bigcup_{i=1}^{N} D_{i}^{\prime}
\end{aligned}
$$

Then we compute $T_{\text {init }}=2.69 T_{\text {rebuf }}=0, F_{\text {rebuf }}=0$ as an example. According to formula 11, we get ${ }^{\prime} A^{\prime}=B^{\prime}=C^{\prime}=\left[\begin{array}{lllll}1 & 0 & 0 & \ldots .\end{array}\right]_{1 \times 40}$

$$
\begin{aligned}
& R_{1 A}=A_{1} \rightarrow D_{1}= \\
& A_{P B} \rightarrow D_{P B}=\left[\begin{array}{c}
1.0 \\
0.88 \\
0.79 \\
0.72 \\
\cdot \\
0.01
\end{array}\right]_{40 \times 1} \wedge\left[\begin{array}{llllll}
0.0 & 0.0 & 0.0 & . & 0.5 & 1.0
\end{array}\right]_{1 \times 8} \\
& =\left[\begin{array}{cccccc}
0.0 & 0.0 & \cdot & \cdot & 0.5 & 1.0 \\
0.0 & 0.0 & \cdot & . & 0.5 & 0.88 \\
0.0 & 0.0 & . & . & 0.5 & 0.79 \\
0.0 & 0.0 & . & \cdot & 0.5 & 0.72 \\
\cdot & \cdot & \cdot & \cdot & . & \cdot \\
0.0 & 0.0 & . & . & 0.01 & 0.01
\end{array}\right]_{40 \times 8} \\
& D_{1 A}^{\prime}=A^{\prime} \circ\left(A_{1} \rightarrow D_{1}\right)=\left[\begin{array}{lllll}
1 & 0 & 0 & 0 & \ldots .0
\end{array}\right]_{1 \times 40} \circ R_{1 A}=\left[\begin{array}{lllll}
0 & \ldots & 0 & 0.5 & 1
\end{array}\right]_{1 \times 8}
\end{aligned}
$$

In the same way we can compute $D_{1 B}^{\prime}, D^{\prime}$.

$D_{1}^{\prime}=D_{1 A}^{\prime} \cap D_{1 B}^{\prime} \cap D_{1 C}^{\prime}=\left[\begin{array}{lllllll}0 & 0 & 0 & \ldots & 0 & 0.5 & 1\end{array}\right]_{1 \times 8}$

According to Table 3, we can get :

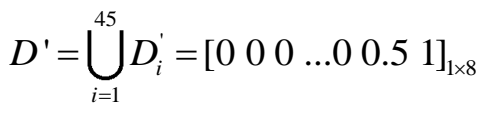

At last we average the D'.

$$
D_{0}=d f\left(D^{\prime}\right)=\frac{0 \times 1+\ldots+0.5 \times 4.5+1 \times 5}{0+\ldots+0.5+1}=4.83
$$

The corresponding subjective MOS is 4.92 . The objective and subjective are similarity. 


\section{The Experimental Results}

In the same way, we can compute all the objective MOS. We also perform the same studies on the other three video sequences. There are 160 times compute. Here we compare with the [10] and [13]'s method. The [10] provides the assessment method:

MOS=4.23-0.0672Tinit-0.742(Frebuf/60)-0.106Trebuf

[10] changes the application metrics into three different levels. According to table 4 Tint $=2.69$, Trebuf $=0$, Frebuf $=0$ can be expressed 2, 1, 1 (Low represents 1, Medium represents 2, High represents 3). So the formula 13 can get the MOS=4. It is different from the subjective score.

\section{Table 4. The Different Level of Application Metrics}

\begin{tabular}{llll}
\hline \multirow{2}{*}{ Level } & \multicolumn{3}{c}{ APMs } \\
\cline { 2 - 4 } & $T_{\text {init }}$ & $f_{\text {rebuf }}$ & $T_{\text {rebuf }}$ \\
\hline Low & $0-1$ seconds & $0-0.02$ & $0-5$ seconds \\
Medium & $1-5$ seconds & $0.02-0.15$ & $5-10$ seconds \\
High & $>5$ seconds & $>0.15$ & $>10$ seconds \\
\hline
\end{tabular}

The [13] provides the assessment method:

$$
f_{3}\left(F_{\text {rebuf }}\right)=a \cdot e^{b \cdot F_{\text {rebuf }}}+c
$$

We also use the formula 14 to regression the objective results. According to table 5, the experimental results show that our proposed method accords closely with human subjective judgment, and improve the similarity. The correlation coefficients are above 0.90 for every video sequence.

Table 5. The Experimental Results

\begin{tabular}{|l|l|l|l|l|}
\hline Method & RMSE & Pearson & Spearman & OR \\
\hline $\begin{array}{l}\text { Fuzzy } \\
\text { inference }\end{array}$ & 0.062 & 0.92 & 0.90 & 0.23 \\
\hline $\begin{array}{l}\text { Liner } \\
\text { regression }\end{array}$ & 0.17 & 0.80 & 0.78 & 0.63 \\
\hline $\begin{array}{l}\text { Exponential } \\
\text { regression }\end{array}$ & 0.13 & 0.83 & 0.81 & 0.57 \\
\hline
\end{tabular}

\section{Conclusions}

In this paper we propose a temporal evaluation of video as the application QoS for HTTP video streaming. We use a set of application QoS for study: Initial buffering time, mean duration of a re-buffering event and re-buffering frequency. Based on these experiment data, we correlate the relationship between the application QoS and the user QoE using fuzzy math method. This method can improve the similarity of subjective and objective method. 


\section{References}

[1] Kuschnig.R,“ Improving Internet Video Streaming Performance by Parallel TCP-Based RequestResponse Streams" Consumer Communications and Networking Conference(CCNC), Klagenfurt,Austria,pp.1-5,9-12 Jan.2010.

[2] Adobe.Video technology center, delivery: Progressive download. http://www.adobe.com/devnet/video/ progressive.html.

[3] N.Staelens, S.Moens,"Assessing quality of experience of IPTV and video on demand services in reallife environments," IEEE Trans. Broadcast., vol.56,no.4,pp.458-466,Sep.2010.

[4] Kalpana Seshadrinathan,Rajiv Soundararajan,Alan Conrad Bovik, et al .Study of Subjective and Objective Quality Assessment of Video[J].IEEE Trans on Image Processing, 2010,19(6):1427-1441.

[5] Stefan Winkler, Praveen Mohandas. The Evolution of Video Quality Measurement: From PSNR to Hybrid Metrics[J].IEEE Transactions on Broadcasting,2008,54(3):660-668.

[6] T.Kim and M.Ammar.Receiver buffer requirement for video streaming over TCP. In Proc, SPIE, pages p.422-431,2006

[7] B.Wang, J.Kurose,P.Shenoy,et al.Multimedia streaming via TCP: An analytic performance study. ACM Trans.Multimedia Comput.Commun.Appl.,4(2):1-22,2008.

[8] Fuzheng Yang. No-Reference Quality Assessment for Networked video via Primary Analysis of Bit Stream[J].IEEE Transactions on,2010,20(11):1544-1554.

[9] O.Verscheure, P.Frossard, and M.Hamdi.MPEG-2 video services over packet networks: Joint effect of encoding rate. In Proc.NOSSDAV, 1998.

[10] Mok,Ricky K.P.,et al.Measuring the Quality of Experience of HTTP Video Streaming[C]//IM 2011,Dublin:Ireland,2011:485-492.

[11] Methodology for the Subjective Assessment of the Quality of Television Pictures, ITU-R Recommendation BT.500-11.

[12] Shiyuan Yang. A new diagnosis approach for handling tolerance in analog and mixed-signal circuits by using fuzzy math[J].IEEE Transactions on Circuits and Systems, 2005,52(10):2118-2127.

[13] Egger,S,et al.Waiting times in quality of experience for web based services, Quality of Multimedia Experience(QoMEX),2012 Fourth International workshop on,5-7 July,2012, Yarry Valley,VIC,86-96.

[14] Singh,K.D,et al.Quality of experience estimation for adaptive HTTP/TCP video streaming using H.264/AVC,2012 IEEE Consumer Communications and Networking Conference(CCNC),14-17 Jan 2012,Las Vegas,NV,127-131. 